\title{
Sample maturation calculated using Raman spectroscopic parameters for solid organics: Methodology and geological applications
}

\author{
LIU DeHan ${ }^{*}$, XIAO XianMing ${ }^{*}$, TIAN Hui, MIN YuShun, ZHOU Qin, CHENG Peng \& \\ SHEN JiaGui
}

State Key Laboratory of Organic Geochemistry, Guangzhou Institute of Geochemistry, Chinese Academy of Sciences, Guangzhou 510640, China

Received August 1, 2012; accepted October 10, 2012; published online December 11, 2012

\begin{abstract}
The shape and shift of Raman peak of solid organics prove to be capable of revealing atomic and molecular level vibration information of aromatic ring structure and its relationship with sample maturation. Raman " $D$ " peaks and "G" peaks of a series of continuously maturated coal samples were measured, and the inter-peak intervals (G-D) and peak height ratios (Dh/Gh) were derived and correlated with the vitrinite reflectance $\left(v R_{0} \%\right)$ of standard coal samples. As a result, two formulae were established by using the two Raman indices for calculation of Raman reflectance $\left(R m c R_{0} \%\right)$, which is equivalent to vitrinite reflectance. The formula for calculating Raman reflectance indicative of organic maturation using Raman shift inter-peak interval $(\mathrm{G}-\mathrm{D})$ is $R m c R_{0} \%=$ $0.0537 d(\mathrm{G}-\mathrm{D})-11.21$, which is mainly applicable to matured to highly matured carbonized samples of solid organics; The formula for calculating Raman reflectance indicative of organic maturation using Raman peak height ratio $(\mathrm{Dh} / \mathrm{Gh})$ is $R m c R_{0} \%=$ $1.1659 h(\mathrm{Dh} / \mathrm{Gh})+2.7588$, which is mainly applicable to carbonized samples of solid organics that are over matured or going to be turned into granulated graphite. Preliminary applications indicate that Raman reflectance " $R m c R_{o} \%$ " calculated based on results of Raman spectral analysis of solid organics can be used to characterize sample maturation at molecular level, so enjoying extensive prospects in geological applications.
\end{abstract}

micro-laser Raman spectroscopic analysis, Raman shift peak $D$ and peak $G$, solid organics, vitrinite reflectance $\left(v R_{0} \%\right)$, Raman index indicative of maturation of solid organics $\left(\operatorname{RmcR}_{\mathbf{0}} \%\right)$

Citation: Liu D H, Xiao X M, Tian H, et al. Sample maturation calculated using Raman spectroscopic parameters for solid organics: Methodology and geological applications. Chin Sci Bull, 2013, 58: 1285-1298, doi: 10.1007/s11434-012-5535-y

In petroleum geology and coal petrography, vitrinite reflectance $\left(v R_{\mathrm{o}} \%\right)$ is a generally universal index gauging maturation of hydrocarbon source rocks and coals. However, highly matured to over-matured samples would show extensive variations in vitrinite reflectance due to considerable inhomogeneity, which would affect accurate assessment of sample maturation. Recently, peak D and peak G in Raman scattering of carbon nanotubes and natural solid organics seem to reflect not only structures and performance of carbon nanotubes but also thermal evolution of carboniferous solid organics in geological samples, and temperature and pressure conditions for experimental samples, so attracting extensive attention from the academic communities in the

*Corresponding authors (email: Liudh@gig.ac.cn; mxiao@gig.ac.cn) world. For example, Ferrari and Robertson [1], Yang and $\mathrm{Wu}$ [2] and Gao et al. [3] reported applications of Raman peaks of carbon in analysis and research of structures and performance of carbon nanotube materials; $\mathrm{Hu}$ and Wilkins [4] as well as Hu et al. [5] reported applications of Raman spectra of solid organics as geothermometers, and found that Raman "G-D" inter-peak interval is decreased while "G/D" peak height ratio is increased when carboniferous sedimentary and metamorphic rocks show Raman reflectance varying from $2.1 \%$ to $14 \%$; Kelemen and Fang [6] as well as Zeng and $\mathrm{Wu}$ [7] reported the relationships between Raman inter-peak interval (D-G) and peak height ratio (D/G) of pyrolytic products of bitumite and kerogen and maturation of samples, or the temperature and pressure conditions for experimental samples. These authors concluded that 
Raman spectra for carbonized substances can be used to assess maturation of organics from catagenesis stage to metamorphic stage, but failed to produce detailed indices for such assessment; Schopf and Kudryavtsev [8], Schopf et al. [9], Schopf et al. [10], and Schopf et al. [11] studied extensively the Raman peak D and peak G characteristics for carbonized microfossils in Precambrian strata, and based on Raman peak shift and peak intensity, calculated Raman index of preservation (RIP), geological age and maturation grade of microfossils in geological samples, and the results proves to be significant for study of origin and evolution of microfossils in Precambrian strata. However, the Raman index of preservation (RIP) thus calculated is not well correlated to vitrinite reflectance that reflects organic maturation, thus its application is rather limited in petroleum geology.

Information on molecular vibration as revealed in Raman spectra of solid organics may be applied as index gauging maturation of geological samples. By referring to the dynamics equation-based Easy $R_{\mathrm{o}} \%$ model as proposed by Sweeney and Burnham [12], we suggest the vitrinite reflectance-equivalent $R_{m} m R_{\mathrm{o}} \%$ model and related methodology based on Raman spectral indices. We measured the Raman spectra of industrial standard coal samples, analyzed the Raman inter-peak interval (G-D) and peak height ratio (Dh/ $\mathrm{Gh}$ ), and studied their variations among different matured samples and their relationships with vitrinite reflectance $\left(v R_{\mathrm{o}} \%\right)$. Based on a large group of data, the formula for calculation of Raman reflectance $\left(R m c R_{\mathrm{o}} \%\right)$ corresponding to vitrinite reflectance $\left(v R_{\mathrm{o}} \%\right)$ was derived using Raman indices, and applied to calculation of Raman reflectance $\left(R m c R_{\mathrm{o}} \%\right)$ for other naturally carbonized solid organic samples. As vitrinite reflectance of solid organics in tiny samples, trace samples or mineral inclusions is hard to be derived using conventional optical methods, and maturation of solid organics in highly matured coal or solid bitumen is hard to be assessed accurately due to high optical inhomogeneity, this method proves to be useful and helpful.

\section{Samples and experimental conditions}

Samples for this study include standard coal samples of different maturation collected from coal mines and drill core samples of solid organics collected from geological profiles and boreholes. Metamorphic coal series samples range from gas coal, anthracite to super-anthracite; Solid bitumen series samples cover gilsonite, pyrobitumen, anthraxolite, and partly, highly matured kerogen. Micro-laser Raman spectroscopic analysis of samples is focused on small area, characterized by trace amount, and is itself non-destructive, and occurs in situ. Polished sections, untreated solid organics blocks, and even milligram-scale powder samples can all be used.

HORIBA-JY LabRAM fully automatic micro-laser Raman spectroscope and HORIBA-JY Xplora delicate-type multi-functional fully automatic micro-laser Raman spectroscope were used for Raman spectroscopic measurement of major samples and for correlation of test results. Main experimental conditions for Raman analysis include: solid laser device $532 \mathrm{~nm} / 30-50 \mathrm{~mW}$, laser energy attenuation D1-D2, raster 1800 lines, confocal pinhole 100-300 $\mu \mathrm{m}$, raster slit $100 \mu \mathrm{m}$, observation objective $\times 50-\times 100$, exposure time 10-40 s, scanning wave number range 100-4000 $\mathrm{cm}^{-1}$, and silicon wafer to be used for wave number calibration of Raman spectroscope. Raman spectral parameters are to be calculated using the spectral analytical software coming as attachment with the spectroscope. Optical reflectance of vitrinite of coal samples is to be measured using $3 \mathrm{Y}$ Leica DMR XP microphotometer, in accordance with the coal petrographic stipulations. Main experimental conditions are listed as follows: yttrium aluminum garnet YAG08-57 $\left(R_{\mathrm{O}}=0.904 \%\right)$, NR1149 $\left(R_{\mathrm{o}}=1.24 \%\right)$ and cubic zirconia $\left(R_{0}=3.11 \%\right)$ will be used, together with immersion oil $(N=1.515)$, measurement objective $50 \times / 0.85$ Oil, optical fiber $d=0.6 \mathrm{~mm}$, based on sample maturation, different standard samples.

\section{Raman spectra of solid organics}

By referring to Yang and $\mathrm{Wu}$ [2] as well as Gao et al. [3], it can be found that Raman spectra of carbon nanotubes consist of four types of Raman peaks: Raman shift at ca. 160$300 \mathrm{~cm}^{-1}$ refers to respiratory vibration mode, mainly reflecting symmetric vibration of carbon atoms in nanotubes; Raman shift at ca. $1250-1450 \mathrm{~cm}^{-1}$, i.e. peak D (generally termed disorder band), refers to dual resonance Raman scattering mode, mainly reflecting information on defects in lattice structures and vacancies in aromatic ring lamellae; Raman shift at ca. $1500-1605 \mathrm{~cm}^{-1}$, i.e. peak G (generally termed order band), mainly refers to the longitudinal stretching vibration mode of $\mathrm{C}=\mathrm{C}$ bond. Raman shift at 2500$2700 \mathrm{~cm}^{-1}$ refers to divalent overtone band $\left(\mathrm{G}^{\prime}\right)[2]$. Shown in Figure 1 is a Raman spectrum of powdered graphite manufactured by Guangzhou Xingang Chemical Engineering Co. Ltd., which clearly shows the Raman shift values for peak " $D$ ", peak " $G$ " and the divalent overtone $\left(G^{\prime}\right)$. He et al. [13], Zhang et al. [14], Zhang et al. [15], and Liu et al. [16] studied the Raman spectra of bitumen and bitumen-bearing hydrocarbon inclusions in oil reservoir, and identified Raman peak D and peak G indicative of bitumen; Liu et al. [17] and Liu et al. [18] studied the high density methane inclusions derived from pyrolysis of oil inclusions in Puguang gas field in Sichuan, and also identified Raman peak $D$ and peak $G$ as well as divalent peak bulge $\left(G^{\prime}\right)$ indicative presence of pyrobitumen in methane inclusions; Zeng and $\mathrm{Wu}$ [7] performed systematic Raman measurement of kerogen simulation samples at 50-100 MPa and 250-700 ${ }^{\circ} \mathrm{C}$, while Kelemen and Fang [6] carried out comprehensive Raman spectroscopic study of coal and kerogen 


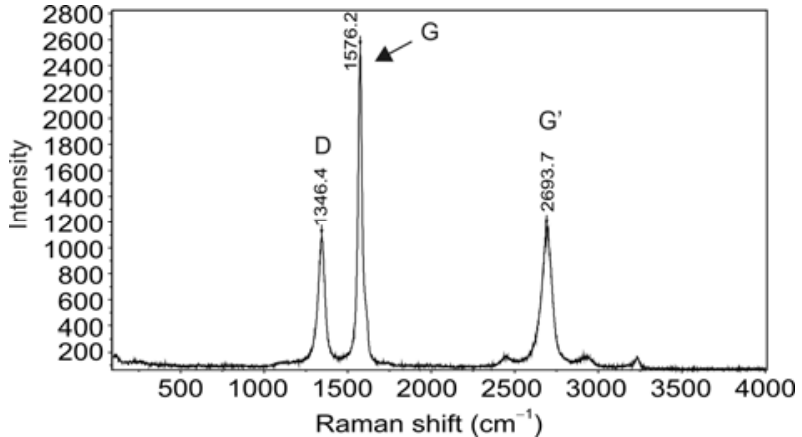

Figure 1 Laser Raman spectra for powdered graphite. Peak D 1346.4 $\mathrm{cm}^{-1}$, peak G $1576.2 \mathrm{~cm}^{-1}$, peak $\mathrm{G}^{\prime} 2693.7 \mathrm{~cm}^{-1}$.
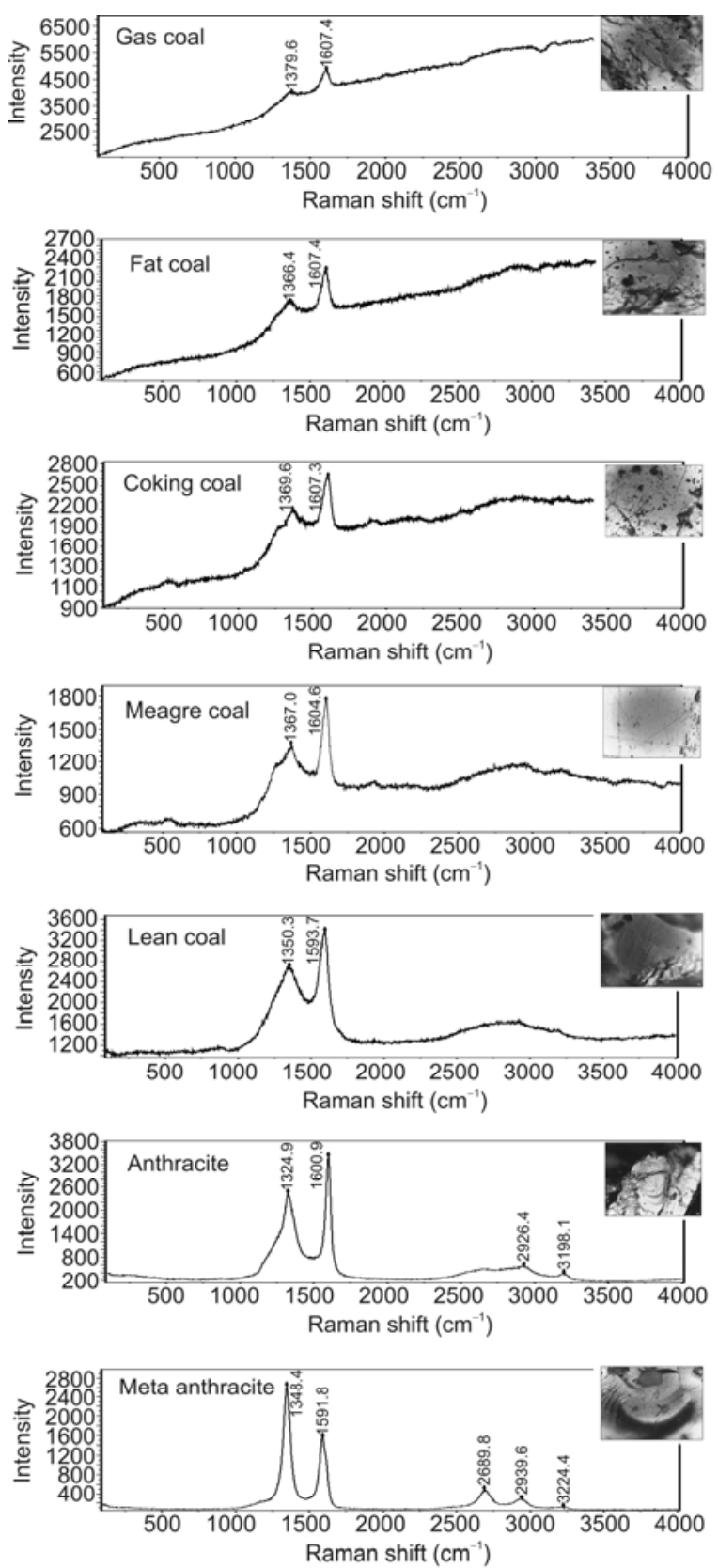

(a) of different maturation, and their results all suggest that: with increasing maturation of samples, peak " $D$ " would shift to lower frequency at ca. $50 \mathrm{~cm}^{-1}$, but to high frequency at not more than $10 \mathrm{~cm}^{-1}$, the inter-peak interval between peak $\mathrm{D}$ and peak $\mathrm{G}$ is closely related to $v R_{\mathrm{o}}$ of experimental sample, and the widths of both peak " $D$ " and peak "G" and the area ratio between these two peaks are all decreased with increasing reflectance.

Figure 2(a) shows Raman spectra of a series of industrial coal grades ranging from gas coal, rich coal, lean coal, meager coal, to anthracite-anthracite, while Figure 2(b) shows Raman spectra of maturated series of solid bitumen
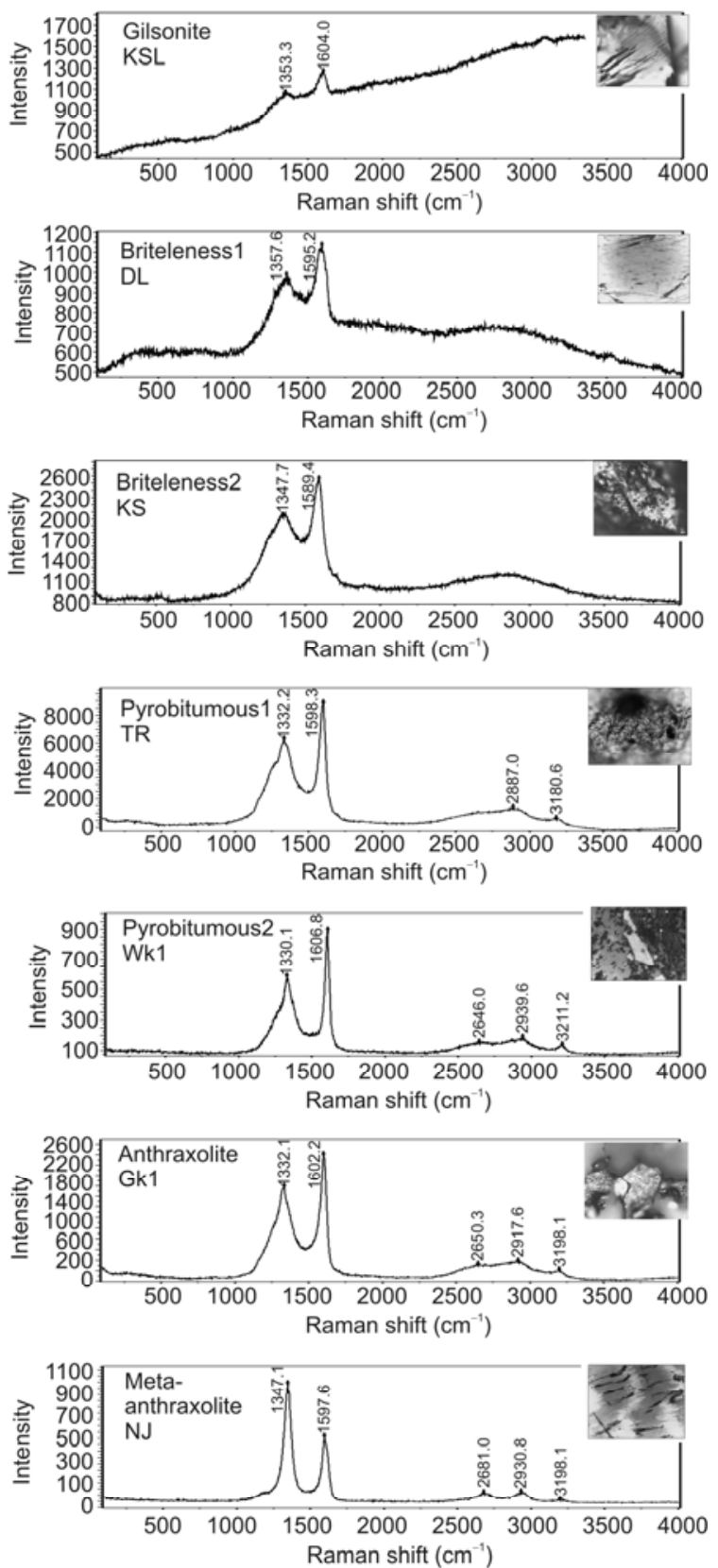

(b)

Figure 2 Raman spectra of maturation series of coal and solid bitumen. 
ranging from gilsonite, grahamite, pyrobitumen, to anthraxolite-meta-exsudatinite. It can be seen that the two maturated series of samples show similar rule of change in Raman spectra. In low maturation stage, Raman spectra generally show very weak, irregularly-shaped and asymmetrical peak $\mathrm{D}$ due to interference from strong fluorescence, in contrast to relatively more remarkable peak G. With increasing maturation, both peak $\mathrm{G}$ and peak become more remarkable, and divalent peak bulge $\left(\mathrm{G}^{\prime}\right)$ appear at ca. $2500-2700 \mathrm{~cm}^{-1}$. At the stage for formation of super-anthracite and metaexsudatinite, Raman peak D>peak G phenomenon would appear.

Further analysis of the Raman spectroscopic parameter changes indicates that: from maturation to high maturation stage, the inter-peak interval between peak " $G$ " and peak "D" in terms of Raman shift (G-D) would increase gradually, and the peak height ratio $(\mathrm{Dh} / \mathrm{Gh})$ would also increase but at much slower pace; From over maturation to graphitization stage, the Raman peak height ratio (Dh/Gh) would increase at faster pace, but the inter-peak interval $(\mathrm{G}-\mathrm{D})$ would decrease instead; At graphitization stage, the divalent peak bulge $\left(\mathrm{G}^{\prime}\right)$ would become stronger, and peak bulge $\left(\mathrm{G}^{\prime}\right)>$ peak "D" phenomenon would appear.

\section{Reflectance calculation using Raman parameters}

As suggested by Raman analytical data for a great amount of samples, Raman spectra of all types of solid organics in nature would show, aside from interference due to strong fluorescence, peak D and peak G, even though of different peak shapes and peak intensity, and with increasing maturation of samples, these peaks would become more remarkable, so would the inter-peak interval and the peak height ratio. In terms of vibration mode of carbon atoms in the aromatic structure of solid organics, all types of solid organics would show maturation progressing toward graphitization. In order to apply Raman analytical results to geology and geochemistry, the Raman parameters indicative of the vibration modes of carbon atoms in the aromatic ring structure of samples would be used to calculate Raman reflectance $\left(R m c R_{\mathrm{o}} \%\right)$, which is equivalent to vitrinite reflectance $\left(v R_{\mathrm{o}} \%\right)$ that can reflect the maturation grade and the surface optical properties of samples. Raman reflectance can be calculated through the following routes: precise measurement of Raman parameters for standard coal samples precisely calibrated with vitrinite reflectance, extensive measurement of vitrinite reflectance and Raman parameters for samples of different maturation grades, and derivation of formula for calculation of Raman reflectance $\left(R m c R_{\mathrm{o}} \%\right)$ which corresponds to and is equivalent to vitrinite reflectance. Since Raman parameters for solid organics would show complicated variations in terms of change rule and mechanism in the process of maturation of samples, the
Raman inter-peak interval (G-D) and peak height ratio (Dh/Gh) would show different mechanisms and change rules for samples at different maturation stages. As a result, we would like to explore the formula for calculation of Raman reflectance applicable to the two following maturation stages:

From maturation to high maturation stage (i.e. from gas coal to anthracite stage), Raman inter-peak interval between peak $G$ and peak $D(G-D)$ would increase linearly with increasing vitrinite reflectance. Therefore, regression can be made between the measured Raman interval $(\mathrm{G}-\mathrm{D})$ data and the vitrinite reflectance $\left(\nu R_{\mathrm{o}} \%\right)$ data for the samples (Figure 3 ), and the formula for calculation of Raman reflectance $\left(R m c R_{\mathrm{o}} \%\right)$ based on the matching relationship between Raman peak interval $(\mathrm{G}-\mathrm{D})$ and vitrinite reflectance $\left(v R_{\mathrm{o}} \%\right)$ can be derived as

$$
R m c R_{\mathrm{o}} \%=0.0537 d(\mathrm{G}-\mathrm{D})-11.21,
$$

where $R m c R_{\mathrm{o}} \%$ refers to Raman reflectance calculated using Raman parameters; (G-D) refers to Raman inter-peak in terms of Raman shift.

From over maturation to super-anthracite stage, the Raman inter-peak interval (G-D) would not increase but decrease instead, since molecular vibration mode changes in aromatic ring structure of solid organics, Raman reflectance at high anthracite stage cannot be calculated simply using Raman inter-peak interval. However, at high anthracite stage, the Raman peak height ratio $(\mathrm{Dh} / \mathrm{Gh})$ as calculated using Raman peak processing software shows remarkable increase with increasing maturation of samples. Therefore, regression can be made between Raman peak height ratio (Dh/Gh) and vitrinite reflectance for the samples (Figure 4), and the formula for calculation of Raman reflectance $R m c R_{\mathrm{O}} \%$ can be derived based on the matching relationship between Raman peak height ratio $(\mathrm{Dh} / \mathrm{Gh})$ and vitrinite reflectance $\left(v R_{0} \%\right)$ as

$$
R m c R_{\mathrm{o}} \%=1.1659 h(\mathrm{Dh} / \mathrm{Gh})+2.7588,
$$

where $R m c R_{\mathrm{o}} \%$ refers to Raman reflectance using Raman

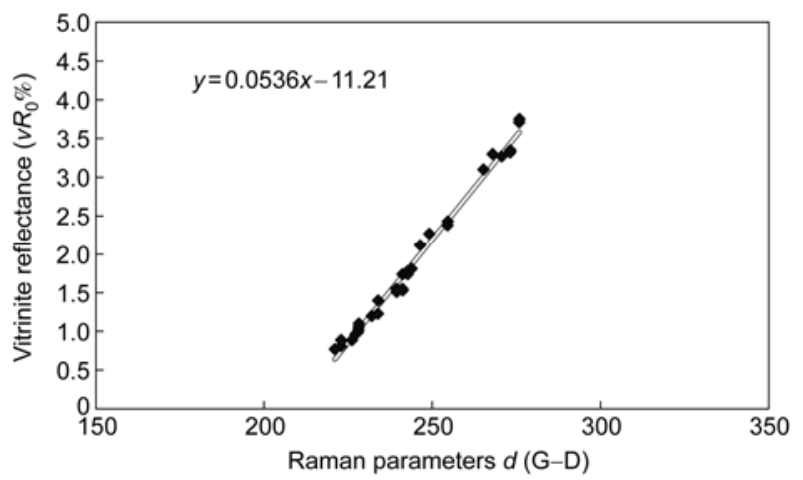

Figure 3 Raman spectra and modal curves of $d(\mathrm{G}-\mathrm{D})$ vs. $v R_{0} \%$ for vitrinite in matured to highly matured coal samples. 


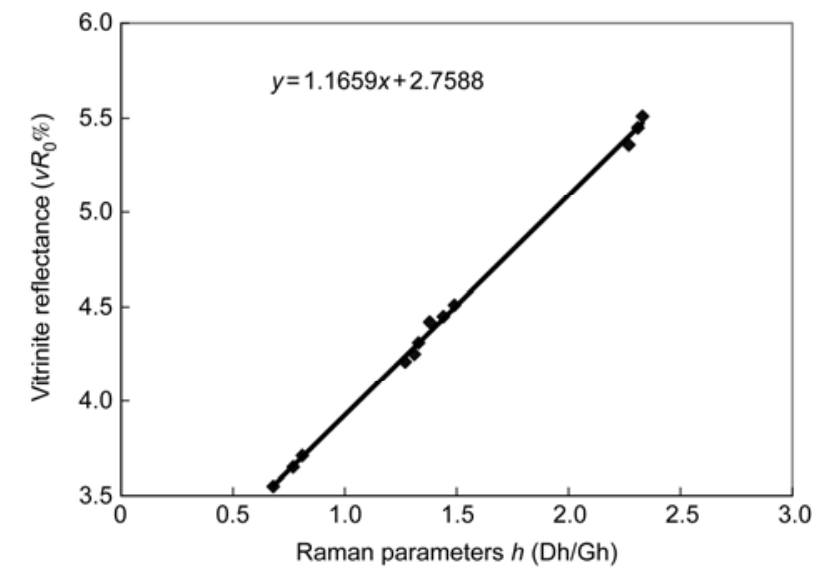

Figure 4 Raman spectra and modal curves of $h(\mathrm{Dh} / \mathrm{Gh})$ vs. $v R_{\mathrm{o}} \%$ for vitrinite in highly matured to over-matured coal samples.

parameters; $h(\mathrm{Dh} / \mathrm{Gh})$ refers to Raman peak height ratio (Dh refers to peak D height, Gh refers to peak $\mathrm{G}$ height).

The two formulae as mentioned above can be used to derive maturation index from Raman peak $D$ and peak $G$, which reflect the carbon atomic vibration in aromatic ring structures of solid organics, and the maturation grade thus derived is correlated well with the vitrinite reflectance grade that reflects the surface optical properties of solid organics samples. Therefore, for all kinds of carbonized solid organics samples related to sedimentation or diagenesis, whenever Raman peak D and peak $G$ which reveal carbon atomic vibration in aromatic ring structure of solid organics appear in their Raman spectra, the Raman reflectance calculation formula can be applied to calculation of maturation of solid organics in these samples. For example, for kerogen, solid bitumen, micronite, carbonized fossils of both animals and plants and solid organics in mineral inclusions, their Raman parameters can be used to calculate the Raman reflectance $\left(R m c R_{\mathrm{o}} \%\right)$ of samples. The precision and application scope of Raman reflectance is related to sample maturation. At low maturation stage, Raman peak D is weak and tend to be subject to interference from strong fluorescence of samples, so is hard to be precisely calibrated, which would affect the precision of Raman reflectance derived; When maturated sample shows vitrinite reflectance being above $1.5 \%$, Raman peak D would show enhanced intensity and sharpness due to reduced interference from fluorescence of samples, which would increase the precision of calculated Raman reflectance. Additionally, for lowly matured samples, Raman peak height treated with Raman processing software package would show relatively low precision; for highly matured to over matured samples, Raman spectra can be well repeated and Raman peak would show enhanced precision, which would facilitate precise measurement and calculation of Raman reflectance of these samples. However, when samples show thermal metamorphism to such a high degree that their Raman peaks show $\left(G^{\prime}\right)>D$, these samples have entered particulate graphitization stage, their carbon atomic vibration mode undergoes abrupt changes, and as a result, the two formulae as mentioned above can not be applied to calculation of reflectance of these samples.

Table 1 lists Raman spectral data, Raman reflectance $\left(R m c R_{\mathrm{o}} \%\right)$ derived from Raman inter-peak interval $(\mathrm{G}-\mathrm{D})$,

Table 1 Raman parameters and correlation between Raman reflectance and vitrinite reflectance for matured to highly matured samples

\begin{tabular}{|c|c|c|c|c|c|c|c|c|}
\hline Sample & $\begin{array}{c}\text { Raman } \\
\text { peak }\end{array}$ & $\begin{array}{c}\text { Raman shift } \\
\left(\mathrm{cm}^{-1}\right)\end{array}$ & $\begin{array}{c}\text { Inter-peak interval } \\
d(\mathrm{G}-\mathrm{D}) \\
\end{array}$ & Peak height & FWHM & $\begin{array}{c}\text { Peak area } \\
\text { (A) }\end{array}$ & $\begin{array}{c}\text { Vitrinite reflectance } \\
\left(v R_{\mathrm{o}} \%\right)\end{array}$ & $\begin{array}{c}\text { Raman reflectance } \\
\left(R m c R_{\mathrm{o}} \%\right)\end{array}$ \\
\hline \multirow{2}{*}{ Gas coal 1} & $\mathrm{D}$ & 1381.07 & \multirow{2}{*}{222.79} & 509.28 & 48.21 & 12014 & \multirow{2}{*}{$0.65-0.71$} & \multirow{2}{*}{0.754} \\
\hline & G & 1603.86 & & 1594.53 & 53.57 & 41788 & & \\
\hline \multirow{2}{*}{ Gas coal 2} & $\mathrm{D}$ & 1381.07 & \multirow{2}{*}{222.61} & 658.44 & 117.85 & 39395 & \multirow{2}{*}{$0.66-0.75$} & \multirow{2}{*}{0.744} \\
\hline & G & 1603.68 & & 135.27 & 53.57 & 49405 & & \\
\hline \multirow{2}{*}{ Rich coal 1} & $\mathrm{D}$ & 1375.28 & \multirow{2}{*}{227.96} & 3834.27 & 262.51 & 480796 & \multirow{2}{*}{$0.91-1.08$} & \multirow{2}{*}{1.03} \\
\hline & G & 1603.24 & & 6287.49 & 64.28 & 197514 & & \\
\hline \multirow{2}{*}{ Rich coal 2} & & 1370.90 & \multirow{2}{*}{228.44} & 2072.99 & 96.43 & 97283 & \multirow{2}{*}{$0.95-1.20$} & \multirow{2}{*}{1.06} \\
\hline & & 1599.34 & & 5088.60 & 58.93 & 163902 & & \\
\hline \multirow{2}{*}{ Coking coal 1} & $\mathrm{D}$ & 1372.27 & \multirow{2}{*}{233.83} & 2904.98 & 75 & 119940 & \multirow{2}{*}{$1.2-1.40$} & \multirow{2}{*}{1.345} \\
\hline & G & 1606.10 & & 4913.94 & 48.21 & 77144 & & \\
\hline \multirow{2}{*}{ Coking coal 2} & $\mathrm{D}$ & 1372.30 & \multirow{2}{*}{233.80} & 2868.57 & 75 & 104955 & \multirow{2}{*}{$1.25-1.50$} & \multirow{2}{*}{1.345} \\
\hline & G & 1606.10 & & 4830.08 & 48.21 & 11390 & & \\
\hline \multirow{2}{*}{ Coking coal 3} & $\mathrm{D}$ & 1365.60 & \multirow{2}{*}{237.20} & 3178.54 & 69.64 & 108052 & \multirow{2}{*}{$1.5-1.60$} & \multirow{2}{*}{1.52} \\
\hline & G & 1603.10 & & 6593.65 & 53.57 & 172804 & & \\
\hline \multirow{2}{*}{ Lean coal 1} & $\mathrm{D}$ & 1362.87 & \multirow{2}{*}{239.45} & 6342.88 & 214.29 & 652732 & \multirow{2}{*}{$1.65-1.70$} & \multirow{2}{*}{1.65} \\
\hline & G & 1602.32 & & 10267.80 & 53.57 & 269096 & & \\
\hline \multirow{2}{*}{ Lean coal 2} & $\mathrm{D}$ & 1360.61 & \multirow{2}{*}{242.07} & 6732.19 & 241.07 & 77015 & $175-185$ & 170 \\
\hline & G & 1602.68 & & 10522.20 & 53.57 & 275762 & $1.13-1.03$ & 1.19 \\
\hline J ean col 3 & $\mathrm{D}$ & 1355.90 & 24240 & 5921.90 & 214.28 & 609367 & $18-100$ & 181 \\
\hline Lean coal 5 & $\mathrm{G}$ & 1598.30 & 242.40 & 9591.80 & 64.28 & 301300 & $1.8-1.90$ & 1.81 \\
\hline
\end{tabular}


(Continued $)$

\begin{tabular}{|c|c|c|c|c|c|c|c|c|}
\hline Sample & $\begin{array}{c}\text { Raman } \\
\text { peak }\end{array}$ & $\begin{array}{c}\text { Raman shift } \\
\left(\mathrm{cm}^{-1}\right)\end{array}$ & $\begin{array}{c}\text { Inter-peak interval } \\
d(\mathrm{G}-\mathrm{D})\end{array}$ & Peak height & FWHM & $\begin{array}{l}\text { Peak area } \\
\text { (A) }\end{array}$ & $\begin{array}{l}\text { Vitrinite reflectance } \\
\left(v R_{\mathrm{o}} \%\right)\end{array}$ & $\begin{array}{c}\text { Raman reflectance } \\
\left(R m c R_{0} \%\right)\end{array}$ \\
\hline \multirow{2}{*}{ Meager coal 1} & D & 1350.89 & \multirow{2}{*}{249.11} & 1426.56 & 251.23 & 705107 & \multirow{2}{*}{$2.05-2.27$} & \multirow{2}{*}{2.17} \\
\hline & G & 1600 & & 2143.58 & 65.08 & 280553 & & \\
\hline \multirow{2}{*}{ Meager coal 2} & $\mathrm{D}$ & 1353.57 & \multirow{2}{*}{246.43} & 7756.73 & 225 & 837042 & \multirow{2}{*}{$2.05-2.15$} & \multirow{2}{*}{2.02} \\
\hline & G & 1600 & & 12316 & 58.93 & 354855 & & \\
\hline \multirow{2}{*}{ Anthracite1 } & $\mathrm{D}$ & 1346.1 & \multirow{2}{*}{254.5} & 7193.22 & 245.71 & 895460 & \multirow{2}{*}{$2.2-2.45$} & \multirow{2}{*}{2.46} \\
\hline & G & 1600.6 & & 13182.2 & 53.57 & 345475 & & \\
\hline \multirow{2}{*}{ Anthracite2 } & $\mathrm{D}$ & 1345.54 & \multirow{2}{*}{257.14} & 9356.9 & 246.43 & 1103090 & \multirow{2}{*}{$2.3-2.47$} & \multirow{2}{*}{2.60} \\
\hline & G & 1602.68 & & 14763 & 48.12 & 348454 & & \\
\hline \multirow{2}{*}{ Anthracite3 } & $\mathrm{D}$ & 1321.4 & \multirow{2}{*}{275.9} & 3389.48 & 192.86 & 314562 & \multirow{2}{*}{$3.5-3.78$} & \multirow{2}{*}{3.61} \\
\hline & G & 1597.32 & & 4394.5 & 53.57 & 115169 & & \\
\hline \multirow{2}{*}{ Anthracite4 } & $\mathrm{D}$ & 1326.79 & \multirow{2}{*}{275.89} & 11402 & 123.21 & 657584 & \multirow{2}{*}{$3.5-3.78$} & \multirow{2}{*}{3.61} \\
\hline & G & 1602.68 & & 17166 & 42.86 & 360304 & & \\
\hline \multirow{2}{*}{ Super-anthracite } & D & 1346.98 & \multirow{2}{*}{$252.21 *$} & 2272.32 & 66.98 & 360492 & \multirow{2}{*}{$4.2-5.81$} & \multirow{2}{*}{$4.91^{*}$} \\
\hline & G & 1599.19 & & 1231.38 & 58.19 & 138653 & & \\
\hline
\end{tabular}

* At super-anthracite stage, inter-peak interval $d(\mathrm{G}-\mathrm{D})$ decreases, so peak height ratio $h(\mathrm{D} / \mathrm{G})=1.845$ is used instead to derive Raman reflectance $\left(\operatorname{RmcR} R_{0} \%\right)=4.91 \%$.

and vitrinite reflectance $\left(v R_{\mathrm{o}}\right)$ of matured to highly matured coal samples. Here vitrinite reflectance $\left(v R_{0} \%\right)$ shows its variation range, Raman reflectance $\left(R m c R_{\mathrm{o}} \%\right)$ is calculated using Raman parameters.

Table 2 lists Raman spectral data, Raman reflectance $\left(R m c R_{\mathrm{o}} \%\right)$ derived using Raman inter-peak interval (G-D) of lowly matured gilsonite and highly matured pyrobitumen and anthraxolite, as well as bitumen reflectance. Bitumen optical reflectance $\left(b R_{\mathrm{o}} \%\right)$ shows its variation range, while Raman reflectance $\left(R m c R_{\mathrm{o}} \%\right)$ is measured using Raman parameters as listed in Table 2.

Table 3 lists Raman spectral data, Raman reflectance $\left(R m c R_{\mathrm{o}} \%\right)$ derived using Raman inter-peak interval $(\mathrm{G}-\mathrm{D})$ and vitrinite reflectance $\left(v R_{0}\right)$ of matured anthracite and highly matured anthraxolite. In Table 3, highly matured samples would show strong inhomogeneity, which would cause variations of measured optical reflectance data, so Raman reflectance $\left(R m c R_{0} \%\right)$ data derived using Raman inter-peak interval $(\mathrm{G}-\mathrm{D})$ can better reflect the maturation of samples.

\section{Influence of sample maceral and optical inhomogeneity on Raman reflectance calculation}

\subsection{Influence of sample maceral on Raman reflectance calculation}

Raman reflectance $\left(R m c R_{\mathrm{o}} \%\right)$ as derived using Raman peak "D" and peak "G" parameters for solid organics can reflect structural changes of carbonized substances in thermal maturation. In lowly matured stage, the carbonized substances in vitrinite, semi-vitrinite, semi-fusinite, fusinite or inertinite in coal maceral would show different aromatic ring polycondensation, which would lead to remarkable differences in Raman reflectance $\left(R m c R_{\mathrm{o}} \%\right.$ ) calculated (Table 4$)$. Therefore, Raman spectroscopic analysis should be focused on vitrinite. However, with increasing maturation, samples would show gradually narrowing differences in maceral. In regard to solid organics such as bitumen characterized by homogeneous maceral distribution, Raman spectrum measured at each spot and Raman reflectance $\left(R m c R_{\mathrm{o}} \%\right)$ calculated using Raman parameters would be similar to each other.

\subsection{Influence of sample optical inhomogeneity on Raman reflectance calculation}

Highly matured anthracite, particularly highly matured pyrobitumen, anthraxolite, and so on, would show strong optical inhomogeneity and remarkable double reflection, therefore, optical reflectance data measured shall be marked "maximum reflectance" $\left(R_{\max }^{\mathrm{o}} \%\right)$, "average reflectance" $\left(R_{\text {min }}^{\mathrm{o}} \%\right)$ or "random reflectance" $\left(R_{\text {ren }}^{\mathrm{o}} \%\right)$. In some highly matured anthracite-super-anthracite, vitrinite would show maximum reflectance $\left(R_{\text {max }}^{\mathrm{o}}\right)$ being up to $7.5 \%-8.2 \%$, and minimum reflectance $\left(R_{\text {min }}^{\mathrm{o}}\right)$ is as low as $4.2 \%-4.5 \%$. Results of Raman spectral analysis can basically reveal microscopic information concerning molecular vibration inside samples, so will not be subject to influence of macroscopic orientations of samples, and as a result, the Raman spectrum measured at each spot of a sample and Raman reflectance $\left(R m c R_{\mathrm{o}} \%\right)$ calculated using Raman parameters would be basically similar to each other for the whole sample.

For pyrobitumen-anthraxolite, their microscopic reflection photomicrographs (Figure 5) show strong double reflection, as optical reflectance can be up to $6.7 \%$ at bright direction but becomes $3.4 \%$ at dark direction (Figure 5(b)); 
Table 2 Raman parameters and correlation between Raman reflectance and vitrinite reflectance for solid bitumen samples

\begin{tabular}{|c|c|c|c|c|c|c|c|c|c|}
\hline Sample & $\begin{array}{l}\text { Raman } \\
\text { peak }\end{array}$ & $\begin{array}{l}\text { Raman shift } \\
\left(\mathrm{cm}^{-1}\right)\end{array}$ & $\begin{array}{c}\text { Inter-peak } \\
\text { interval } \\
\text { (G-D) } \\
\end{array}$ & Peak height & FWHM & $\begin{array}{l}\text { Peak area } \\
\text { (A) }\end{array}$ & $\begin{array}{c}\text { Bitumen } \\
\text { reflectance } \\
\left(b R_{0} \%\right)\end{array}$ & $\begin{array}{c}\text { Raman } \\
\text { reflectance } \\
\left(R m c R_{0} \%\right)\end{array}$ & Remarks \\
\hline $\begin{array}{l}\text { Gilsonite } \\
\text { K6 }\end{array}$ & $\begin{array}{l}\mathrm{D} \\
\mathrm{G}\end{array}$ & $\begin{array}{l}1369.62 \\
1587.73\end{array}$ & 222.85 & $\begin{array}{l}2106.38 \\
2340.46\end{array}$ & $\begin{array}{r}267.28 \\
86.62\end{array}$ & $\begin{array}{l}912439 \\
273981\end{array}$ & $0.6-0.74$ & 0.754 & $\begin{array}{l}\text { Not remarkable peak D due } \\
\text { to intense fluorescence }\end{array}$ \\
\hline $\begin{array}{l}\text { gilsonite } \\
\text { K-S-L }\end{array}$ & $\begin{array}{l}\mathrm{D} \\
\mathrm{G}\end{array}$ & $\begin{array}{l}1360.9 \\
1583.19\end{array}$ & 222.29 & $\begin{array}{l}2488.63 \\
2948.71\end{array}$ & $\begin{array}{l}254.18 \\
107.55\end{array}$ & $\begin{array}{l}10310.6 \\
52247.2\end{array}$ & $0.6-0.68$ & 0.727 & $\begin{array}{l}\text { Not remarkable peak D due } \\
\text { to intense fluorescence }\end{array}$ \\
\hline $\begin{array}{l}\text { Dalong } \\
\text { DL }\end{array}$ & $\begin{array}{l}\mathrm{D} \\
\mathrm{G}\end{array}$ & $\begin{array}{l}1355.21 \\
1591.18\end{array}$ & 235.97 & $\begin{array}{r}980.24 \\
1163.29\end{array}$ & $\begin{array}{c}226.5 \\
97.63\end{array}$ & $\begin{array}{l}438157 \\
227606\end{array}$ & $1.2-1.6$ & 1.46 & $\begin{array}{l}\text { Subject to interference } \\
\text { from fluorescence }\end{array}$ \\
\hline $\begin{array}{l}\text { Ludian } \\
\mathrm{Cu}\end{array}$ & $\begin{array}{l}\mathrm{D} \\
\mathrm{G}\end{array}$ & $\begin{array}{l}1357.58 \\
1600\end{array}$ & 242.4 & $\begin{array}{l}7002.78 \\
11601.6\end{array}$ & $\begin{array}{r}203.57 \\
53.57\end{array}$ & $\begin{array}{l}685405 \\
304052\end{array}$ & $1.7-2.2$ & 1.80 & Bitumen in copper mine \\
\hline $\begin{array}{l}\text { Kangshan } 1 \\
\text { K-S }\end{array}$ & $\begin{array}{l}D \\
G\end{array}$ & $\begin{array}{l}1356.25 \\
1597.32\end{array}$ & 242.07 & $\begin{array}{r}7074.7 \\
10633.3\end{array}$ & $\begin{array}{c}262.5 \\
64.28\end{array}$ & $\begin{array}{l}886923 \\
334035\end{array}$ & $1.8-2.4$ & 1.79 & Inhomogeneous bitumen \\
\hline $\begin{array}{l}\text { Kangshan } 2 \\
\text { KoSa }\end{array}$ & $\begin{array}{l}\mathrm{D} \\
\mathrm{G}\end{array}$ & $\begin{array}{l}1358.0 \\
1599.8\end{array}$ & 241.8 & $\begin{array}{l}7075.28 \\
9474.38\end{array}$ & $\begin{array}{r}267.06 \\
58.93\end{array}$ & $\begin{array}{l}904566 \\
272979\end{array}$ & $1.8-2.2$ & 1.77 & Inhomogeneous bitumen \\
\hline $\begin{array}{l}\text { Kangshan } 3 \\
\text { K-S }\end{array}$ & $\begin{array}{l}\mathrm{D} \\
\mathrm{G}\end{array}$ & $\begin{array}{l}1355.42 \\
1602.66\end{array}$ & 247.24 & $\begin{array}{l}8545.22 \\
11749.8\end{array}$ & $\begin{array}{c}262.5 \\
58.93\end{array}$ & $\begin{array}{r}1671260 \\
330762\end{array}$ & $1.9-2.5$ & 2.07 & Inhomogeneous bitumen \\
\hline $\begin{array}{l}\text { Tongren } \mathrm{CHg} \\
\text { H-S-D }\end{array}$ & $\begin{array}{l}\mathrm{D} \\
\mathrm{G}\end{array}$ & $\begin{array}{l}1332.3 \\
1601\end{array}$ & 268.7 & $\begin{array}{l}9289.81 \\
15748.1\end{array}$ & $\begin{array}{r}208.93 \\
48.21\end{array}$ & $\begin{array}{l}932379 \\
371656\end{array}$ & $3.1-3.3$ & 3.10 & $\begin{array}{l}\text { Intergrowth between py- } \\
\text { robitumen and cinnabar }\end{array}$ \\
\hline $\begin{array}{l}\text { Wanshan } € \\
\text { Hg-WS }\end{array}$ & $\begin{array}{l}\mathrm{D} \\
\mathrm{G}\end{array}$ & $\begin{array}{l}1337.5 \\
1602.7\end{array}$ & 265.2 & $\begin{array}{l}9289.21 \\
157481\end{array}$ & $\begin{array}{r}208.93 \\
48.21\end{array}$ & $\begin{array}{l}932379 \\
371656\end{array}$ & $3.2-3.5$ & 3.23 & $\begin{array}{l}\text { Intergrowth between py- } \\
\text { robitumen and cinnabar }\end{array}$ \\
\hline $\begin{array}{l}\text { Danzhai } € \\
\text { Hg- D-Z }\end{array}$ & $\begin{array}{l}D \\
G\end{array}$ & $\begin{array}{l}1329.4 \\
1602.68\end{array}$ & 268.7 & $\begin{array}{l}1709.78 \\
2142.32\end{array}$ & $\begin{array}{r}123.21 \\
42.86\end{array}$ & $\begin{array}{r}102153 \\
44966\end{array}$ & $3.5-4.2$ & 3.46 & $\begin{array}{l}\text { Intergrowth between py- } \\
\text { robitumen and cinnabar }\end{array}$ \\
\hline $\begin{array}{l}\text { Puguang gas field } \\
\text { PG3- }\end{array}$ & $\begin{array}{l}\mathrm{D} \\
\mathrm{G}\end{array}$ & $\begin{array}{l}1356.0 \\
1610.4\end{array}$ & 254.4 & $\begin{array}{l}2783.29 \\
5429.61\end{array}$ & $\begin{array}{r}198.21 \\
42.86\end{array}$ & $\begin{array}{l}265405 \\
113965\end{array}$ & $2.1-2.6$ & 2.48 & Homogeneous \\
\hline $\begin{array}{l}\text { Puguang gas field } \\
\text { PG5-c }\end{array}$ & $\begin{array}{l}\mathrm{D} \\
\mathrm{G}\end{array}$ & $\begin{array}{l}1330.7 \\
1602.6\end{array}$ & 271.9 & $\begin{array}{l}5810.09 \\
7875.29\end{array}$ & $\begin{array}{r}166.07 \\
53.57\end{array}$ & $\begin{array}{l}465785 \\
206393\end{array}$ & $3.2-3.5$ & 3.39 & $\begin{array}{l}\text { Inhomogeneous pyrobitu- } \\
\text { men }\end{array}$ \\
\hline $\begin{array}{l}\text { Chuandong S1 } \\
\text { WK1 }\end{array}$ & $\begin{array}{l}\mathrm{D} \\
\mathrm{G}\end{array}$ & $\begin{array}{l}1327.52 \\
1600.58\end{array}$ & 273.06 & $\begin{array}{l}1958.65 \\
2701.04\end{array}$ & $\begin{array}{r}124.91 \\
37.76\end{array}$ & $\begin{array}{l}420246 \\
182307\end{array}$ & $3.3-3.6$ & 3.45 & $\begin{array}{l}\text { Inhomogeneous pyrobitu- } \\
\text { men }\end{array}$ \\
\hline $\begin{array}{l}\text { Chuanzhong Z } \\
\text { GK1-3 }\end{array}$ & $\begin{array}{l}\mathrm{D} \\
\mathrm{G}\end{array}$ & $\begin{array}{l}1326.79 \\
1602.68\end{array}$ & 275.89 & $\begin{array}{l}8604.68 \\
12537.3\end{array}$ & $\begin{array}{r}166.07 \\
48.21\end{array}$ & $\begin{array}{l}689800 \\
295880\end{array}$ & $3.4-3.8$ & 3.605 & $\begin{array}{l}\text { Remarkably inhomogene- } \\
\text { ous anthraxolite }\end{array}$ \\
\hline $\begin{array}{l}\text { Weiyuan W4-3-5 } \\
\text { Z }\end{array}$ & $\begin{array}{l}\mathrm{D} \\
\mathrm{G}\end{array}$ & $\begin{array}{l}1329.45 \\
1608.04\end{array}$ & 278.59 & $\begin{array}{l}3842.59 \\
5358.71\end{array}$ & $\begin{array}{l}150 \\
48.21\end{array}$ & $\begin{array}{l}278751 \\
126466\end{array}$ & $3.5-3.9$ & 3.75 & $\begin{array}{l}\text { Remarkably inhomogene- } \\
\text { ous anthraxolite }\end{array}$ \\
\hline $\begin{array}{l}\text { Shuitian Z } \\
\text { S-T }\end{array}$ & $\begin{array}{l}\mathrm{D} \\
\mathrm{G}\end{array}$ & $\begin{array}{l}1326.79 \\
1605.36\end{array}$ & 278.61 & $\begin{array}{l}3515.32 \\
4148.87\end{array}$ & $\begin{array}{c}112.5 \\
42.83\end{array}$ & $\begin{array}{r}192074 \\
87076\end{array}$ & $4.5-5.1$ & 3.751 & $\begin{array}{l}\text { Remarkably inhomogene- } \\
\text { ous anthraxolite }\end{array}$ \\
\hline
\end{tabular}

Table 3 Raman parameters and correlation between Raman reflectance and vitrinite reflectance for over-matured samples

\begin{tabular}{|c|c|c|c|c|c|c|c|c|}
\hline Sample & Peak mark & Shift $\left(\mathrm{cm}^{-1}\right)$ & Peak height & $\begin{array}{c}\text { Peak height ratio } \\
(\mathrm{D} / \mathrm{G})\end{array}$ & FWHM & $\begin{array}{l}\text { Peak area } \\
\text { (A) }\end{array}$ & $\begin{array}{c}\text { Vitrinite reflectance } \\
\left(R_{0} \%\right)\end{array}$ & $\begin{array}{c}\text { Raman reflectance } \\
\left(R m c R_{0} \%\right)\end{array}$ \\
\hline \multirow{2}{*}{ Anthraxolite Shu-Ta } & $\mathrm{D}$ & 1334.82 & 1770.76 & \multirow[b]{2}{*}{1.04} & 96.43 & 83086.3 & \multirow[b]{2}{*}{$3.5-4.2$} & \multirow[b]{2}{*}{3.97} \\
\hline & G & 1602.68 & 1698.06 & & 53.57 & 44502.3 & & \\
\hline \multirow{2}{*}{$\begin{array}{l}\text { High-grade anthra- } \\
\text { cite Z-Q-D1a }\end{array}$} & D & 1337.5 & 18385.1 & \multirow{2}{*}{1.27} & 91.07 & 815229 & \multirow{2}{*}{$4.02-4.3$} & \multirow{2}{*}{4.24} \\
\hline & G & 1594.64 & 14466.2 & & 58.93 & 416803 & & \\
\hline \multirow{2}{*}{$\begin{array}{l}\text { High-grade anthra- } \\
\text { cite Z-Q-D6 }\end{array}$} & $\mathrm{D}$ & 1342.68 & 15214.9 & \multirow{2}{*}{1.30} & 80.36 & 516019 & \multirow{2}{*}{$4.1-4.37$} & \multirow{2}{*}{4.27} \\
\hline & G & 1594.64 & 11691.4 & & 58.93 & 336855 & & \\
\hline \multirow{2}{*}{$\begin{array}{l}\text { High-grade anthra- } \\
\text { cite Z-Q-D3 }\end{array}$} & $\mathrm{D}$ & 1341.7 & 21051.7 & \multirow{2}{*}{1.33} & 75.0 & 770154 & \multirow{2}{*}{$4.1-4.5$} & \multirow{2}{*}{4.31} \\
\hline & G & 1592.1 & 15865.9 & & 53.51 & 415804 & & \\
\hline \multirow{2}{*}{ Anthraxolite S-Lin2 } & $\mathrm{D}$ & 1342.68 & 7117.87 & \multirow{2}{*}{1.38} & 85.71 & 284469 & \multirow{2}{*}{$4.1-4.5$} & \multirow{2}{*}{4.37} \\
\hline & G & 1600 & 5138.17 & & 64.29 & 139188 & & \\
\hline \multirow{2}{*}{ Anthraxolite Lv Ka2 } & $\mathrm{D}$ & 1353.57 & 2322.1 & \multirow{2}{*}{1.44} & 69.64 & 78934.5 & \multirow{2}{*}{$3.9-4.6$} & \multirow{2}{*}{4.44} \\
\hline & G & 1600 & 1610.34 & & 59.93 & 46397.8 & & \\
\hline \multirow{2}{*}{ Anthraxolite Lv Ka1 } & $\mathrm{D}$ & 1350.89 & 687.53 & \multirow{2}{*}{1.52} & 69.64 & 23370.6 & \multirow{2}{*}{$3.8-4.7$} & \multirow{2}{*}{4.53} \\
\hline & G & 1602.68 & 452.05 & & 53.57 & 11847.3 & & \\
\hline \multirow{2}{*}{$\begin{array}{l}\text { High-grade anthra- } \\
\text { citeZ-Q-D7 }\end{array}$} & D & 1342.86 & 23063.5 & \multirow{2}{*}{2.27} & 58.93 & 664152 & \multirow{2}{*}{$5.2-5.36$} & \multirow{2}{*}{5.41} \\
\hline & G & 1598.29 & 10255.4 & & 53.57 & 266145 & & \\
\hline High-grade anthra- & $\mathrm{D}$ & 1345.51 & 23720.6 & 2326 & 53.57 & 621355 & $53-57$ & 5.47 \\
\hline citeZ-Q-D10 & G & 1589.29 & 10198.7 & & 53.57 & 267281 & & $3.4 /$ \\
\hline High-grade anthra- & $\mathrm{D}$ & 1342.86 & 20180.3 & 231 & 58.93 & 604162 & $521-54$ & 5.45 \\
\hline citeZ-Q-D9 & G & 1583.92 & 9072.86 & 2.01 & 53.57 & 237775 & $5.21-5.4$ & $0.4 \mathrm{~J}$ \\
\hline High-grade anthra- & $\mathrm{D}$ & 1342.86 & 22615.6 & & 58.93 & 651254 & $53-5.7$ & 5.48 \\
\hline citeZ-Q-D7 & G & 1589.29 & 9705.82 & 2.33 & 53.57 & 254364 & $5.3-5.1$ & 5.48 \\
\hline High-grade anthra- & $\mathrm{D}$ & 1342.68 & 20583.7 & 2.37 & 53.57 & 539184 & $5.4-5.81$ & 5.52 \\
\hline citeZ-Q-D9b & $\mathrm{G}$ & 1598.92 & 8675.97 & $2.3 \%$ & 53.57 & 227374 & & \\
\hline
\end{tabular}


Table 4 Calculated Raman reflectance for maceral at coking coal stage

\begin{tabular}{|c|c|c|c|c|c|c|c|c|c|}
\hline No. & Sample & $\begin{array}{l}\text { Peak } \\
\text { mark }\end{array}$ & Shift $\left(\mathrm{cm}^{-1}\right)$ & Peak height & $\begin{array}{c}\text { Inter-peak } \\
\text { interval (G-D) }\end{array}$ & FWHM & $\begin{array}{l}\text { Peak area } \\
\text { (A) }\end{array}$ & Maceral & $\begin{array}{c}\text { Raman reflectance } \\
\left(R m c R_{0} \%\right)\end{array}$ \\
\hline \multirow{2}{*}{1} & \multirow{2}{*}{ Gao Yang (coking coal) } & $\mathrm{D}$ & 1365.6 & 5921.9 & \multirow{2}{*}{237.2} & 69.64 & 108052 & \multirow{2}{*}{ vitrinite } & \multirow{2}{*}{1.53} \\
\hline & & G & 1603.1 & 9591.8 & & 53.57 & 172804 & & \\
\hline \multirow{2}{*}{2} & \multirow{2}{*}{ Gao Yang (coking coal) } & $\mathrm{D}$ & 1361.5 & 4588.83 & \multirow{2}{*}{241.2} & 182.14 & 402848 & \multirow{2}{*}{ semi-vitrinite } & \multirow{2}{*}{1.74} \\
\hline & & G & 1603.7 & 7484.9 & & 53.57 & 196162 & & \\
\hline \multirow{2}{*}{3} & \multirow{2}{*}{ Gao Yang (coking coal) } & $\mathrm{D}$ & 1334.8 & 5459.1 & \multirow{2}{*}{265.72} & 139.29 & 368193 & \multirow{2}{*}{ macrosome (inertinite) } & \multirow{2}{*}{3.06} \\
\hline & & G & 1600.0 & 8652.35 & & 48.21 & 204195 & & \\
\hline \multirow{2}{*}{4} & \multirow{2}{*}{ Gao Yang (coking coal) } & $\mathrm{D}$ & 1337.5 & 4643.83 & \multirow{2}{*}{267.9} & 116.07 & 372309 & \multirow{2}{*}{ fusinite } & \multirow{2}{*}{3.17} \\
\hline & & G & 1605.4 & 6562.91 & & 48.21 & 154885 & & \\
\hline
\end{tabular}
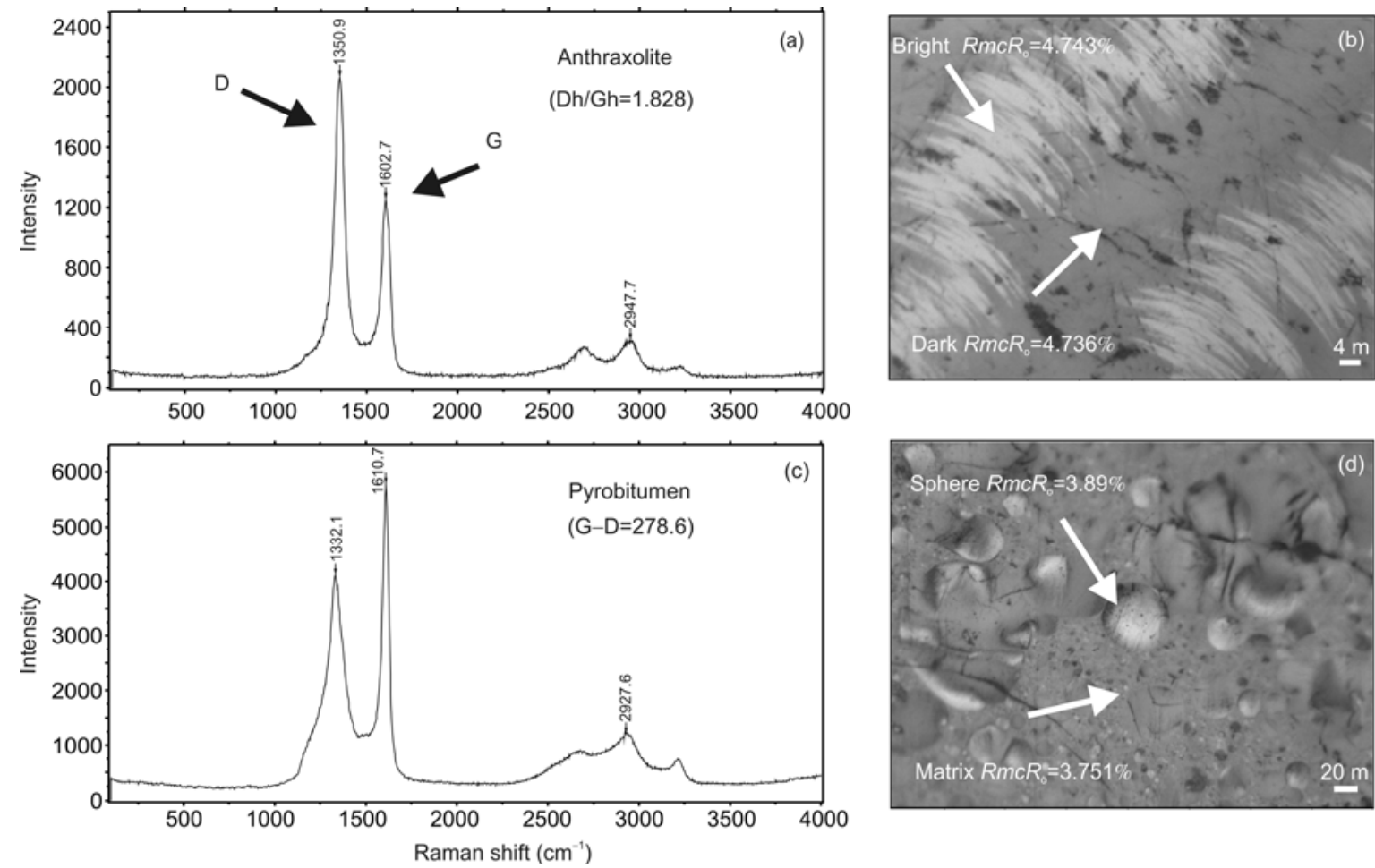

Figure 5 Raman spectra and calculated Raman reflectance data for inhomogeneous anthraxolite and pyrobitumen. Raman spectrum for highly matured anthraxolite; (b) strongly inhomogeneous anthraxolite, showing evenly distributed $R m c R_{0} \%$, photomicrograph in reflection mode, $\times 1000$; (c) Raman spectrum of pyrobitumen with intermediate phase structure; (d) inhomogeneous pyrobitumen with intermediate phase structure, showing evenly distributed $R m c R_{0} \%$, photomicrograph in reflection mode, $\times 1000$.

The bright pyrobitumen spheroid shows optical reflectance of $5.2 \%$ while the dark pyrobitumen spheroid shows optical reflectance $2.8 \%$. However, the Raman spectra and Raman reflectance $\left(R m c R_{\mathrm{o}}\right)$ show little difference between two directions (Figure 5(a)-(d)). Figure 5(b) shows $R m c R_{0}=4.743 \%$ at bright direction but $R m c R_{0}=4.736 \%$ at dark direction; while in Figure 5(d), among pyrobitumen of intermediate phase structure, the bright and big spheroid shows Raman reflectance $\left(R m c R_{\mathrm{o}}\right)$ of $3.89 \%$, the dark and small spheroid shows basically similar Raman spectrum and Raman reflectance $\left(R m c R_{0}=3.751 \%\right)$, even though exhibiting remarkably different optical performance. These results fully demonstrate the significance for assessment of optically inhomogeneous solid organics maturation at high maturation stage by using Raman spectroscopic measurement and Raman reflectance parameters.

\section{Applications of Raman reflectance in geology}

Since laser beam spot used for Raman reflectance measurement is at micrometer scale, laser shows special functions for being able to penetrate to some distance while not destroying sample structures, Raman reflectance $\left(R m c R_{\mathrm{o}}\right)$ derived from Raman spectroscopic analysis proves to be useful for assessment of maturation of solid bitumen in not only coal but also in highly matured reservoir, highly matured hydrocarbon source rocks and mineral inclusions. 


\subsection{Shale gas assezssment}

Raman spectrum-derived reflectance can act as maturation index and is suitable for being applied to precise maturation assessment of highly matured to over-matured samples. In China, shale gas exploration targets mainly lower Cambrian, upper Ordovician and lower Silurian black shale of lower Paleozoic, as well as upper Paleozoic carboniferous mudstone. The strata occurring in upper Yangtze plate (like Sichuan Basin), Tarim Basin and lower Yangtze Plate generally show high maturation, but to what extent and to what degree in terms of maturation? Since most solid organics in black shale is very fine-grained and show strong optical inhomogeneity, it is very hard to precisely measure sample maturation by using conventional method. However, the Raman parameter-derived reflectance $\left(R m c R_{\mathrm{o}}\right)$ calculation method, as established in this study, is not vulnerable to sample preparation conditions, so can be effectively applied to assessment of maturation of multiple organic types in highly matured black shale.

For example, multiple types of maceral can be identified, simply based on morphology, in one black graptolite shale block from Qiaokou, Sichuan (Figure 6), then microscopic laser Raman spectroscopy was used to measure these many maceral varieties in a sample, and Raman reflectance was then calculated from the Raman spectroscopic parameters. Figure 6(a)-(f) illustrates representative spectra and photos of some typical samples, with data showing the range of variations. For example, the Raman parameter (G-D) for marine vitrinite in samples ranges between 267.8-267.9, and the calculated Raman reflectance $\left(R m c R_{\mathrm{o}}\right)$ between $3.17 \%-3.176 \%$; the Raman parameter $(\mathrm{G}-\mathrm{D})$ for solid bitumen ranges between 267.8-270.6, and the calculated Raman reflectance $\left(R m c R_{\mathrm{o}}\right)$ between $3.17 \%-3.32 \%$; the Raman parameter (G-D) for micronite ranges between 269.5270.5 , and the calculated Raman reflectance $\left(R m c R_{\mathrm{o}}\right)$ between $3.262 \%-3.315 \%$; the Raman parameter (G-D) for rhabdosome ranges between 267.7-270.5, and the calculated Raman reflectance $\left(R m c R_{\mathrm{o}}\right)$ between $3.17 \%-3.315 \%$; the Raman parameter (G-D) for intergranular organics that was hard to be identified in samples ranges between 265.1270.4 , and the calculated Raman reflectance between $3.17 \%$ $3.31 \%$. These results demonstrate that highly matured black shale still retains macroscopically macermorphologies, and the maturation derived from Raman parameters that reflect internal molecular vibration modes of carbonized solid organics shows little variations, macerals in highly matured black shale shows Raman reflectance $\left(R m c R_{\mathrm{o}}\right)$ ranging between $3.17 \%-3.32 \%$, with the small variations being mainly related to maturation of black shale in the region.

Regional geological data demonstrates that the Raman reflectance $\left(R m c R_{\mathrm{o}}\right)$ is basically comparable in terms of maturation to vitrinite reflectance $\left(v R_{\mathrm{o}} \%\right)$ derived for the Paleozoic strata in the region. Additionally, as indicated by a great many sample measurement results, Raman reflec- tance $\left(R m c R_{\mathrm{o}}\right)$ for black shale in Cambrian and Silurian strata in Sichuan Basin varies generally above 3\%, while $R m c R_{\mathrm{O}}=3.5 \%-3.7 \%$ for part of the black shale and carbonized shale in southern Anhui Province, showing higher maturation, since Raman spectroscopic characteristics for granular graphite appear on part of the shale sheets.

\subsection{Application in simulation of experimental samples}

In hydrocarbon geochemistry, dynamics test for hydrocarbon generated from organics maturation enjoys extensive applications. However, solid organics residue left over from maturation test in gold tube-sealed closed system is scarce and very fine-grained, its vitrinite reflectance is hard to measure using conventional optic method, so is generally replaced by dynamics parameters-derived Easy $R_{0} \%$. Practically, Raman spectroscope can be used to measure this type of tiny and trace samples, and Raman parameters- derived reflectance $\left(R m c R_{\mathrm{o}}\right)$ can be well correlated with vitrinite reflectance.

At the top of Figure 7 is the Raman spectrum of relict carbonized powder from thermodynamics simulation test of kerogen at $480^{\circ} \mathrm{C}$ and $50 \mathrm{MPa}$ by LIAO Zewen and DU Junyan, the powder giving Raman reflectance $R m c R_{\mathrm{o}}=$ $1.737 \%$ calculated from Raman inter-peak interval $d(\mathrm{G}-\mathrm{D})$. At the middle and bottom of Figure 7 are respectively Raman spectra of solid residue from thermal simulation test of Fushun vitrite and Kuangshanliang bitumen in quartz glass tube-sealed system at $600^{\circ} \mathrm{C}$, the residue samples respectively give Raman reflectance $R m c R_{0}=2.74 \%$ and $2.75 \%$ as calculated from Raman inter-peak interval $d(\mathrm{G}-\mathrm{D})$. Our test results indicate that Raman spectra were hard to get due to serious interference from fluorescence for samples at low temperature maturation stage. Highly matured samples show less interference from fluorescence and remarkable peak D and peak G in Raman spectra, which are so remarkable that the Raman parameters can be effectively used to calculate Raman reflectance $\left(R m c R_{\mathrm{o}}\right)$ to calibrate maturation of the test samples.

\subsection{Maturation of carbonized fossils of both animals and plants}

Multiple carbonized animal and plant microfossils occur in trace amount in strata of various geological ages, and the maturation of these microfossils can also be measured and calibrated using Raman spectroscopy. For example, Schopf and Kudryavtsev [8], Schopf et al. [9], Schopf et al. [10], and Schopf et al. [11] measured extensively Raman spectra of carbonized microfossils in Precambrian strata, calculated the Raman index of preservation (RIP) that reflects the geological age and maturation of the microfossils, and divided the fossils into ten grades (1-9). These results prove to be significant to study of structures and origin of microfossils, but not to maturation undergone by carbonized microbody, 

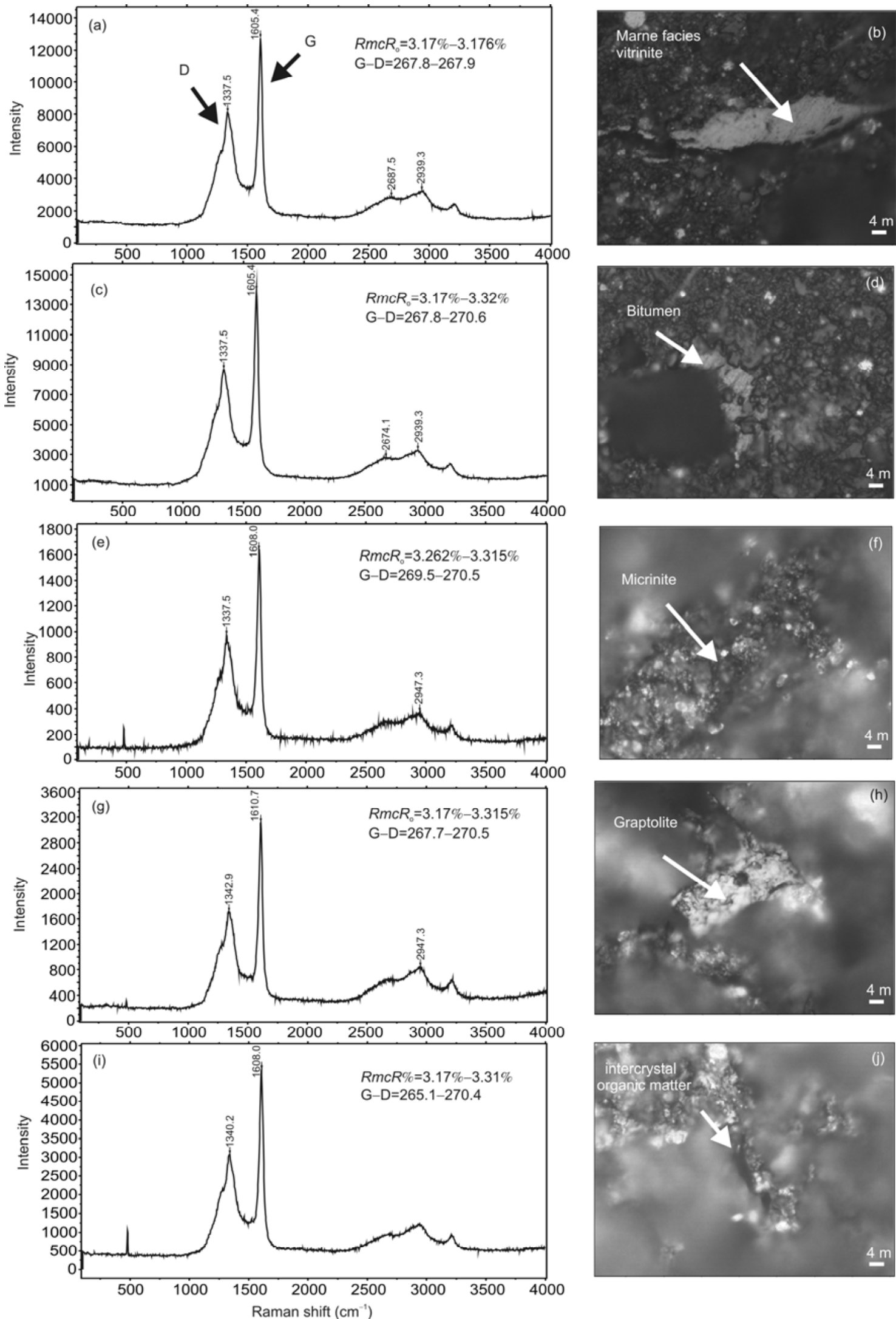

Figure 6 Raman spectra for macerals in black shale. (a), (b) Raman spectrum of marine quasi-vitrinite from lower Silurian black shale, and photomicrograph in reflection mode, $\times 1000$; (c), (d) Raman spectra of solid bitumen, and photomicrographs in reflection mode, $\times 1000$; (e), (f) Raman spectra of micronite, and photomicrographs in reflection mode, $\times 1000$; (g), (f) Raman spectra of rhabdosome, and photomicrographs in reflection mode, $\times 1000$; (i), (j) Raman spectra of intergranular organics, and photomicrographs in reflection mode, $\times 1000$. 

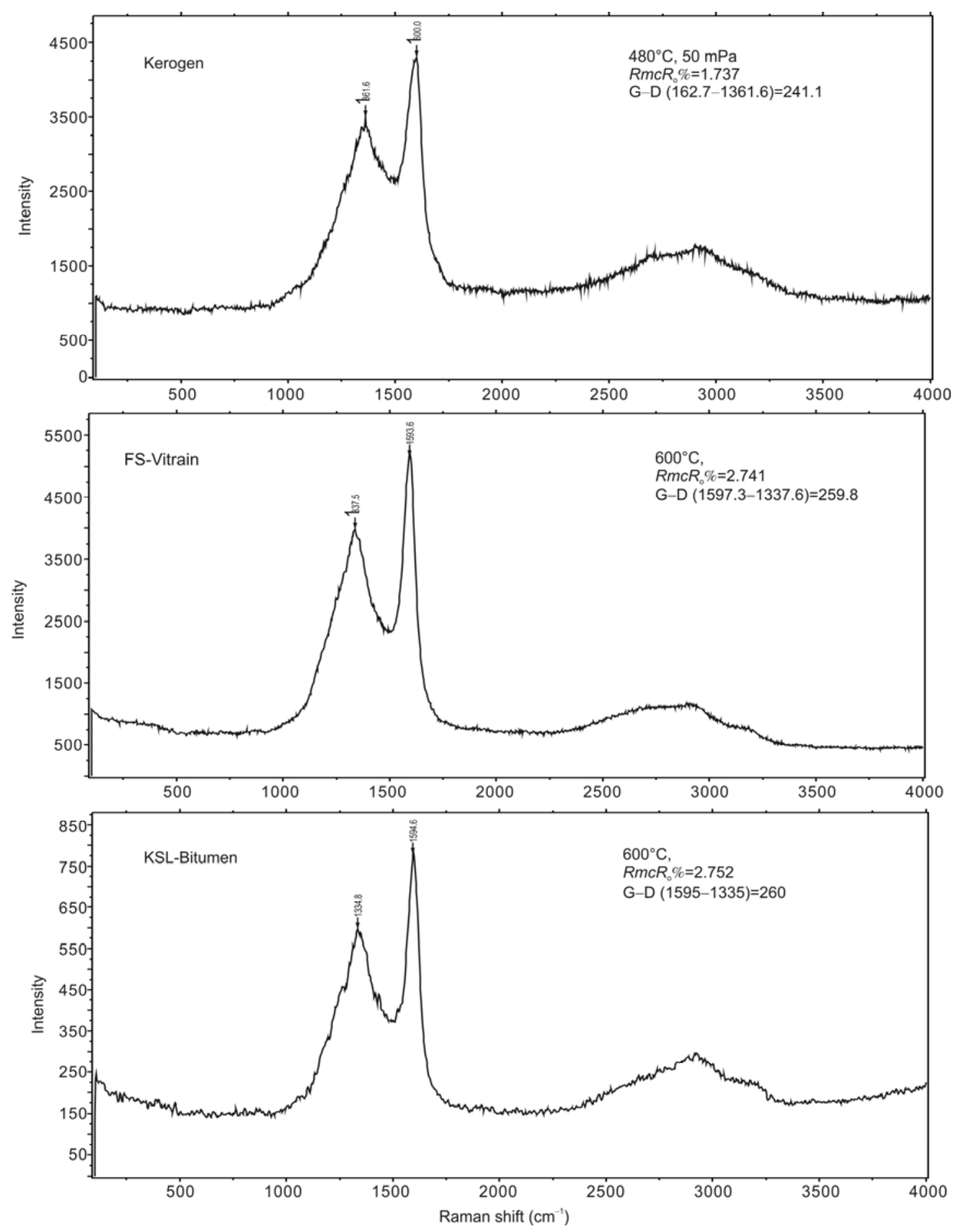

Figure 7 Raman parameter reflectance for thermally simulated test samples. FS: Fushun; KSL: Kuangshanliang.

which can be assessed using thermal maturation indices in organic geochemistry.

Microfossils like cyanophycin are well preserved morphologically in Proterozoic siliceous strata in North China (Figure 8(b),(d)), so can be measured using Raman spectroscope. Figure 8(a)-(c) shows Raman spectra measured for cyanophycin microfossils in polished thin sections of siliceous rock samples, with maturation data calculated from
Raman parameters using methods established in this study. The Raman reflectance $\left(R m c R_{\mathrm{o}}\right)$ is $3.71 \%$ for tubular and silky cyanophycin microfossils, and is $3.83 \%$ for cyanophycin cyst. These results demonstrate that the cyanophycin fossils underwent high maturation even though they were well preserved morphologically in strata.

In addition, carbonized rhabdosome fossils and fragments of well preserved shapes can be frequently discovered in 

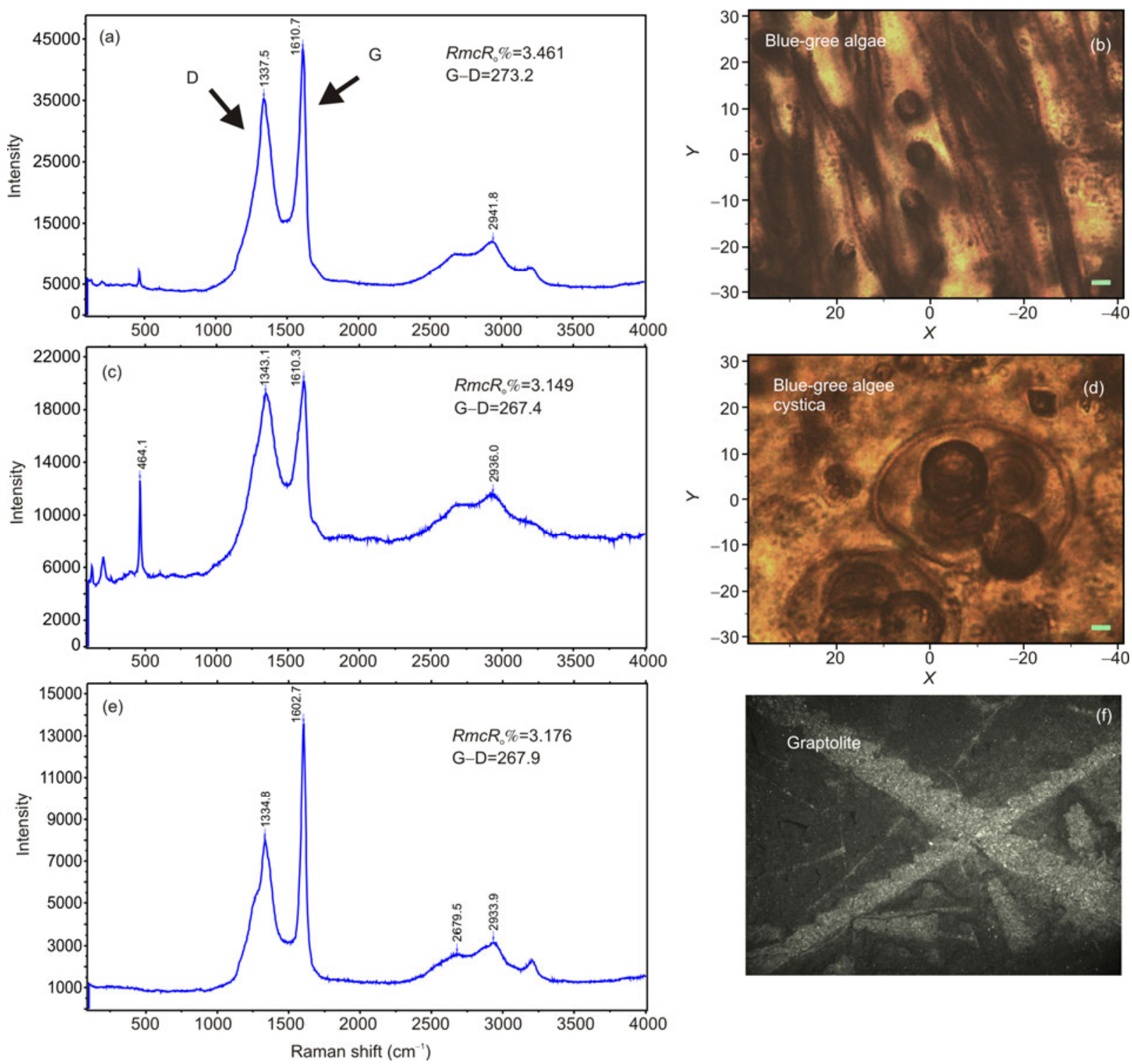

Figure 8 Raman parameter reflectance for Proterozoic Oscillatoriopsis and lower Silurian graptolite. (a), (c), (e) Raman spectra for carbonized microbody and Raman reflectance data; (b), (d) photomicrographs of polished thin sections (used for Raman measurement) of Oscillatoriopsis and its cyst from Proterozoic siliceous rocks, $\times 1000$; (f) photomicrograph of Silurian graptolite shale (used for Raman measurement).

black shale of Silurian and Ordovician strata in lower Paleozoic (Figure 8(f)), so can be measured with Raman spectroscope to derive Raman reflectance $\left(R m c R_{\mathrm{o}} \%\right)$, to be used for assessment of black shale in terms of maturation. Figure 8(e) and (f) shows Raman spectra for rhabdosome in Silurian graptolite shale from Qiaoting, Sichuan, together with Raman reflectance data $R m c R_{0}=3.176 \%$ (Figure 8(e)), which can basically represent maturation of black shale in the geological profile.

\subsection{Maturation of solid organics in mineral inclusions and metallic ore deposits}

Carbonaceous substances occur extensively in minerals and rocks, and mineral inclusions of different origin and differ- ent generations can contain multiple types of organic inclusions. In polished thin sections of these samples, optical reflectance may be measured for some solid organics of large particles, but cannot be measured for solid organics inclusions in minerals. Laser can penetrate into transparent minerals like quartz, so Raman spectra can be measured for tiny organics in minerals, and Raman parameters can be used to calculate Raman reflectance $\left(R m c R_{0} \%\right)$ of this solid organics. Meanwhile, Raman spectra can be measured for gas or liquid components like $\mathrm{CH}_{4}, \mathrm{CO}_{2}, \mathrm{H}_{2} \mathrm{~S}, \mathrm{~N}_{2}, \mathrm{C}_{n} \mathrm{H}_{2 n}$ and so on in mineral inclusions, which can yield key data illuminating the origin and evolution of inclusions and ore deposits.

Raman spectra were measured for solid bitumen-bearing high density methane inclusions in Puguang gas reservoir 

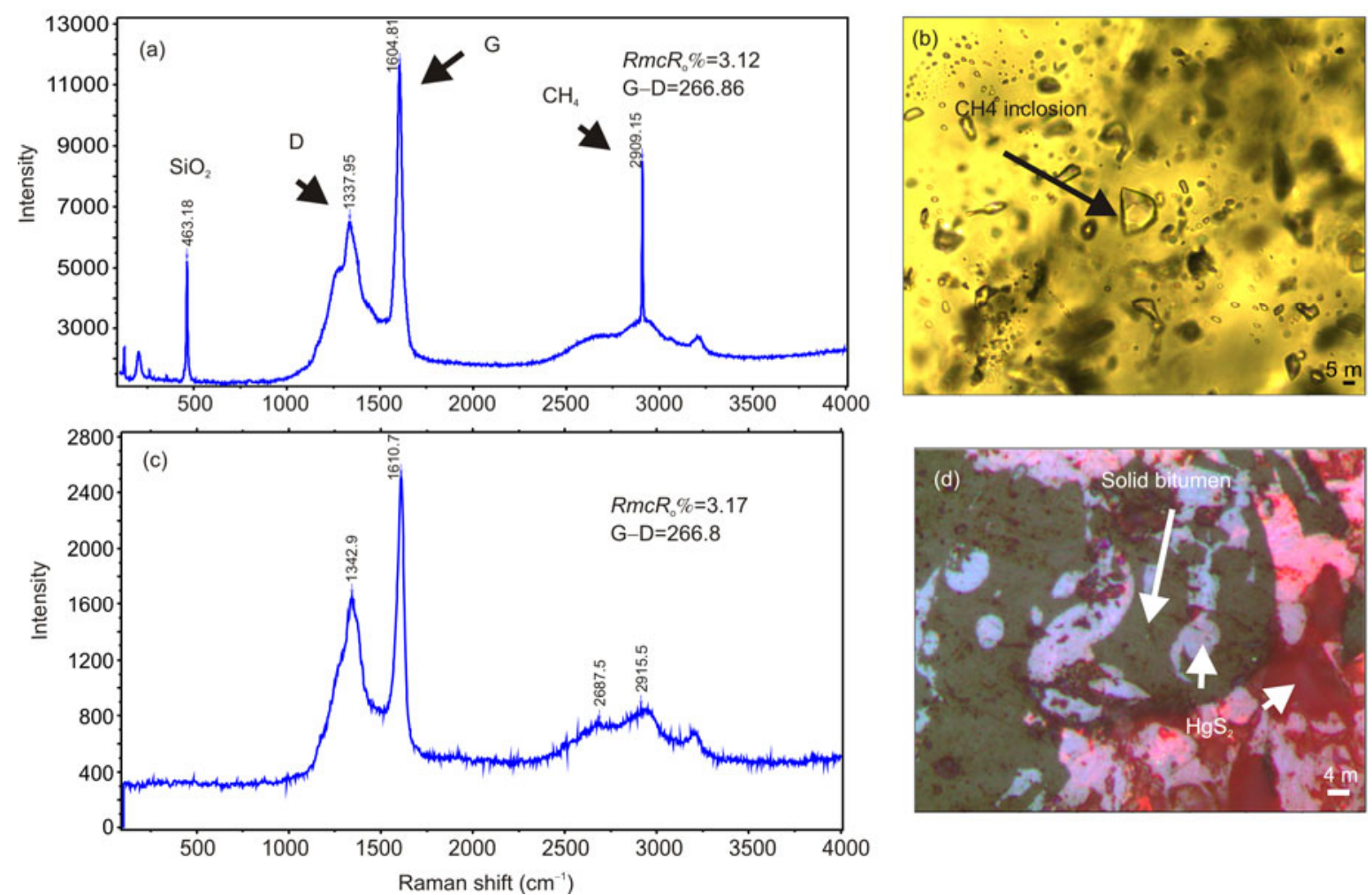

Figure 9 Raman spectra for high density methane inclusions and solid bitumen in mercury mine. (a) Raman spectrum of solid bitumen-bearing high density methane inclusions and Raman reflectance $\left(R m c R_{\mathrm{o}}\right)$; (b) photomicrograph in transmission mode of solid bitumen-bearing high density methane inclusions, used for Raman measurement, $(\times 500)$; (c) Raman spectrum of pyrobitumen in Tongren Cambrian mercury mine; (d) photomicrographs in both reflection and transmission modes of $\mathrm{HgS}_{2}$ in pyrobitumen inclusions, used for Raman measurement.

rocks in Sichuan (Figure 9(a) and (b)). The laser Raman spectra show remarkable peaks $2911-2912 \mathrm{~cm}^{-1}$ characteristic of high density $\mathrm{CH}_{4}$ and some peaks characteristic of $\mathrm{CO}_{2}[17,18]$, apparent peak $\mathrm{D}$ and peak $\mathrm{G}$ characteristic of highly matured solid bitumen, as well as peak $\mathrm{G}^{\prime}$ bulge characteristic of high density methane. Based on Raman spectra shown in Figure 9(a), the Raman reflectance derived is $R m c R_{\mathrm{o}}=3.12 \%$, clearing indicating that this type of inclusions were originated from later stage high temperature cracking of earlier-formed oil inclusions. Raman spectra were also measured for solid bitumen in cinnabar from Wanshan mercury mine in Tongren, Guizhou (Figure 9(c) and (d)), and Raman reflectance can be calculated as $R m c R_{\mathrm{o}}=3.17 \%$, which yields key data for study of the origin of the mercury mine and maturation of the organics in minerals from the mine.

\section{Conclusions}

Raman spectra can be measured for solid organics of various types, as the morphology of Raman peaks, particularly shifts and height ratio for peak D and peak $G$, can fully reflect multiple information concerning molecular vibrations modes of matured carbonized substances, so can be used to assess sample maturation. Raman spectra were measured for a maturated series of coal samples, and the inter-peak interval $d(\mathrm{G}-\mathrm{D})$ and peak height ratio $h(\mathrm{Dh} / \mathrm{Gh})$ were correlated with vitrinite reflectance of the samples. Based on the correlative relationships among the parameters, Raman parameters were used to establish two model equations for calculation of Raman reflectance, which is equivalent to the generally applied parameter, vitrinite reflectance. Model equation (1) describing the relationship between inter-peak interval $d(\mathrm{G}-\mathrm{D})$ and vitrinite reflectance can be well applied to calculation of Raman reflectance $R m c R_{\mathrm{o}} \%$ of matured to highly matured solid organics; model equation (2) describing the relationship between peak height ratio $h(\mathrm{Dh} / \mathrm{Gh})$ and vitrinite reflectance can be well applied to calculation of Raman reflectance $R m c R_{\mathrm{o}} \%$ of over matured to <graphitized solid organics. The maturation index derived from Raman parameters can reflect atomic and molecular vibration modes inside aromatic rings in samples. Our test results demonstrate that the laser Raman spectroscopic analysis of solid organics is not subject to influences such as sample sizes, sample preparation conditions or optical inhomogeneity, so enjoying extensive prospects of application in exploration assessment and geological and geochemical study of hydrocarbon resources. 
Standard coal samples of industrial grades as established by Chinese Academy of Coal Sciences Xi'an Branch were provided by Prof. Chen Deyu, solid bitumen samples were mainly collected from earlier hydrocarbon geological survey funded by Academician Fu Jiamo; Proterozoic silky cyanophycin samples were collected by Prof. Wu Qingyu of Tsinghua University, while intermediate phase pyrobitumen samples were provided by Prof. Sun Yongge of Zhejiang University. The authors would like to extend their thanks to them, and to Profs. Peng Ping'an, Lu Jialan and Jia Rongfen for their help with the research. This work was supported by the National Basic Research Program of China (2012CB214705), the National Natural Science Foundation of China (41072095), the State Scientific Brainstorm Program (2011ZX05008-002) and the Knowledge Innovation Program of the Chinese Academy of Sciences (KZCX2-YW-Q05-03 and $K Z C X 2-Y W-Q 05-06)$.

1 Ferrari A C, Robertson J. Raman Spectroscopy in Carbons: From Carbon Nanotubes to Diamond (in Chinese). Beijing: Chemical Industry Press, 2007

2 Yang X, Wu Q. Raman Spectroscopy Analysis and Application (in Chinese). Beijing: National Defense Industry Press, 2008. 210-243

3 Gao Y, Li L, Tan P, et al. Application of Raman spectroscopy in study of carbon nanotube polymer composite materials (in Chinese). Sci China Earth Sci, 2010, 55: 2165-2176

4 Hu K, Wilkins R W T. Laser Raman spectroscopic carbon geothermometer: A new method for measuring geothermal temperature (in Chinese). Chin Sci Bull (Chin Ver), 1992, 37: 1302-1305

5 Hu K, Liu Y, Wilkins R W T. Raman spectroscopic studies of sedimentary organic matter (in Chinese). Acta Sedimentol Sin, 1993, 11: 64-71

6 Kelemen S R, Fang H L. Maturity trends in Raman spectra from kerogen and coal. Energy Fuels, 2001, 15: 653-658

7 Zeng Y, Wu C. Raman and infrared spectroscopic study of kerogen treated at elevated temperatures and pressures. Fuel, 2007, 86: 1192-
1200

8 Schopf J W, Kudryavtsev A B. Confocal laser scanning microscopy and Raman imagery of ancient microscopic fossils. Precambrian Res, 2009, 173: 39-49

9 Schopf J W, Tewari V, Kudryavtsev A B. Discovery of a new chert-permineralized microbiota in the Proterozoic Buxa Formation of the Ranjit Window, Sikkim, Northeast India, and its astrobiological implications. Astrobiology, 2008, 8: 735-746

10 Schopf J W, Kudryavtsev A B, Czaja A D, et al. Evidence of Archean life: Stromatolites and microfossils. Precambrian Res, 2007, 158: $141-155$

11 Schopf J W, Kudryavtsev A B, Agresti D G, et al. Raman imagery: A new approach to assess the geochemical maturity and biogenicity of permineralized Precambrian fossils. Astrobiology, 2005, 5: 333-371

12 Sweeney J J, Burnham A K. Evolution of a simple model of vitrite reflectance on chemical kinetics. AAPG Bull, 1990, 74: 1559-1570

13 He M, Lü X, Liu Y. Preliminary study of application of laser Raman spectroscopy in hydrocarbon exploration (in Chinese). Spectro Spectral Anal, 2004, 24: 1363-1366

14 Zhang N, Tian Z, Mao G, et al. Raman spectroscopic characteristics of bitumen inclusions (in Chinese). Geochimica, 2009, 38: 174-178

15 Zhang N, Tian Z, Leng Y, et al. Raman spectroscopic characteristics of hydrocarbon and hydrocarbon inclusions (in Chinese). Sci China Ser D: Earth Sci, 2007, 37: 900-907

16 Liu J, Ma C, Wang S, et al. Discovery of solid bitumen inclusions in authigenic rock crystals in Majiang paleo-oil reservoir and its geological significance (in Chinese). Geol Sci Tech Inf, 2008, 28: 39-50

17 Liu D, Dai J, Xiao X, et al. High density methane inclusions in Puguang gas field: Discovery and a T-P genetic study (in Chinese). Chin Sci Bull (Chin Ver), 2009, 54: 4714-4723

18 Liu D H, Xiao X M, Tian H, et al. High density methane inclusions: Evidences for evolution of petroleum and petroleum inclusions and origin $\mathrm{H}_{2} \mathrm{~S}$ (in Chinese). Bull Mineral Petrol Geochem, 2011, 30 (Suppl): 364

Open Access This article is distributed under the terms of the Creative Commons Attribution License which permits any use, distribution, and reproduction in any medium, provided the original author(s) and source are credited. 